\title{
Symplectormophism groups of non-compact manifolds, orbifold balls, and a space of Lagrangians
}

\author{
Richard Hind, Martin Pinsonnault, and Weiwei Wu
}

Let $M=L(n, 1)$ be a 3 -dimensional Lens space. We show that there is a natural map from the loop space of the contact isometry group of $M$ to the compactly supported symplectomorphism group of its symplectization $s M$ which induces a weak homotopy equivalence. We apply this result to determine the topology of a space of symplectic embeddings of orbifold balls and to show that the compactly supported sympectomorphism group of an orbifold ball is contractible. The result also applies to Lagrangian embeddings and we show that the space of Lagrangian $\mathbb{R} P^{2}$ in $T^{*} \mathbb{R} P^{2}$ is contractible.

\section{Introduction}

Since the seminal work of Gromov, [10], the symplectomorphism groups of closed 4-manifolds have been a subject of much research, see for example [1], [20], as have symplectomorphism groups of manifolds with convex ends, see for example [19], [7]. Here we investigate the simplest symplectic manifolds with both convex and concave ends, namely the symplectizations $s L(n, 1)$ of Lens spaces $L(n, 1)$. We show that the compactly supported symplectomorphism group $\operatorname{Symp}_{c}(s L(n, 1))$ has a rich topology. In the first part of the paper we obtain the following result:

Theorem 1.1. The group $\operatorname{Symp}_{c}(s L(n, 1))$, endowed with the $C^{\infty}$-topology, has countably many components, each being weakly homotopy equivalent to the based loop space of $S U(2)$. There is a natural map from $\mathscr{L}\left(\mathcal{C} \mathrm{Iso}_{n}\right)$, the based loop group of contact isometry group of $L(n, 1)$, to $\operatorname{Symp}_{c}(s L(n, 1))$ which induces the weak homotopy equivalence.

Mathematics Subject Classification: 53Dxx, 53D35, 53D12.

Key words and phrases: symplectic packing, symplectomorphism groups, space of Lagrangians, orbifold balls. 
Theorem 1.1 will be proved in Section 2. We start by establishing the weak homotopy equivalence and then construct a natural map from the loop space of contact isometries.

Let us illustrate what is new compared to the cases of closed symplectic manifolds or those with convex ends. Given a contact manifold $M$ with a specified contact form $\alpha$ and $a, b \in[-\infty, \infty]$ with $a<b$ there exists a symplectic structure on $s M_{a}^{b}=M \times(a, b)$ given by $d\left(e^{t} \alpha\right)$, where $t$ is the coordinate on the interval $(a, b)$. A rescaling argument shows that the compactly supported symplectomorphism group of $s M_{a}^{b}$ is weakly homotopic to that of the symplectization $s M$, and so in the case when $M$ is a Lens space $\operatorname{Symp}_{c}\left(s M_{a}^{b}\right)$ has the homotopy type described by Theorem 1.1. On the other hand, if $M$ is embedded in a symplectic manifold $W$ as a contacttype hypersurface then it has a contact form for which $s M_{-\epsilon}^{\epsilon}$ symplectically embeds in $W$ for all $\epsilon$ sufficiently small, and the embedding induces a map between compactly supported symplectomorphism groups. Thus we have a very rich topology of symplectomorphisms supported near $M$. However computations for closed manifolds or manifolds with convex ends give much simpler topology for symplectomorphism groups. Hence the corresponding map on homotopy groups will typically be far from injective. For example, $S^{3} \hookrightarrow B^{4}$ as a contact type hypersurface, but while $\operatorname{Symp}_{c}\left(s S^{3}\right)$ has infinitely many components weakly homotopy equivalent to the based loop space of $U(2)$, it is a result of $[10]$ that $\operatorname{Symp}_{c}\left(B^{4}\right)$ is contractible.

In the second part of the paper we will use the embeddings $s M_{a}^{b} \rightarrow W$ to apply our computations to some natural questions in symplectic topology. This is a fairly general technique which we hope will also be useful elsewhere.

As the first application, in Section 3 we generalize to the orbifold case results of Gromov, [10], saying that the compactly supported symplectomorphism group of a (smooth) symplectic 4-ball is contractible, and McDuff, [16], saying that the space of embeddings of a symplectic 4-ball into a larger 4-ball is homotopic to $U(2)$. We consider singular balls with a conical singularity of order $n \geq 1$ at the origin. The theorem is as follows, which is exactly Gromov's and McDuff's theorems in the smooth case $n=1$.

Theorem 1.2. The space of symplectic embeddings of a singular ball of size $\epsilon \in(0,1)$ into a singular ball of size 1 (with the same singularity of order $n$ ) is homotopy equivalent to the Kähler isometry group $K_{n}$ of the Hirzebruch surface $\mathbb{F}_{n}$. Moreover, the group of reduced, compactly supported symplectomorphisms of a singular ball of size 1 is contractible. 
Here $\mathbb{F}_{n}$ is the Hirzebruch surface $\mathbb{P}(\mathscr{O}(n) \oplus \mathbb{C})$. The the result can be reduced to Theorem 1.1 since on the one hand removing the section at infinity $s_{\infty}$ from $\mathbb{F}_{n}$, and blowing down the zero section $s_{0}$, one obtains a singular 4-ball with a conical singularity of order $n$ at the origin. On the other hand $\mathbb{F}_{n} \backslash\left\{s_{\infty} \cup s_{0}\right\}$ is symplectomorphic to $s L(n, 1)_{-\infty}^{1}$ and so we can determine the compactly supported symplectomorphisms as above.

Finally, in Section 4 we study the space of Lagrangian $\mathbb{R} P^{2}$ in $T^{*} \mathbb{R} P^{2}$ using Theorem 1.1 in the case $n=4$.

Theorem 1.3. The space of Lagrangian $\mathbb{R} P^{2}$ in the cotangent bunble $T^{*} \mathbb{R} P^{2}$, endowed with the $C^{\infty}$-topology, is weakly contractible.

It is already known that the space of Lagrangian $S^{2}$ in $T^{*} S^{2}$ is contractible, see [11], [12]. However this could also be derived from Theorem 1.1 in the case $n=2$.

The proof of Theorem 1.3 involves some additional arguments and also invokes the fact that the diffeomorphism group of $\mathbb{R} P^{2}$ is homotopic to $S O(3)$. We include a proof of this in our Appendix A.

Acknowledgements. The authors would like to thank the MSRI where part of this work was completed. The first author is partially supported by Grant \#317510 from the Simons Foundation. The second author is supported by a NSERC Discovery Grant RGPIN 371999, the third author is supported by NSF Focused Research Grants DMS-0244663.

\section{Symplectomorphism groups of $s L(n, 1)$}

Consider the lens space

$$
L(n, 1)= \begin{cases}S^{3} & n=1 \\ S^{3} / \mathbb{Z}_{n} & n \geq 2\end{cases}
$$

Here, if we think of $S^{3}$ as the unit sphere in $\mathbb{C}^{2}$ then the $\mathbb{Z}_{n}$ action is generated by the diffeomorphism $\left(z_{1}, z_{2}\right) \mapsto\left(e^{2 \pi i / n} z_{1}, e^{2 \pi i / n} z_{2}\right)$. As contact quotients of $S^{3}$ with the standard contact form, the lens spaces inherit natural contact one-forms, denoted as $\lambda_{n}$. There is a standard way to associate a non-compact symplectic manifold to a contact manifold, called the symplectization. Concretely, we consider $L(n, 1) \times \mathbb{R}$ endowed with the symplectic form $d\left(e^{t} \lambda_{n}\right)$, where $t$ is the coordinate of the second factor $\mathbb{R}$. We denote this symplectic manifold $s L(n, 1)$. Compactly supported symplectomorphism groups will be denoted by Symp $_{c}$. In this section, we discuss 
the homotopy type of $\operatorname{Symp}_{c}(s L(n, 1))$, the group of compactly supported symplectomorphisms of $s L(n, 1)$.

\subsection{Reducing $s L(n, 1)$ to compact manifolds}

We first reduce the problem to the symplectomorphism groups of partially compactified symplectic manifolds. Let $\mathscr{O}(n)$ be the complex line bundle over $\mathbb{C} P^{1}$ with Chern class $c_{1}=n$. One can endow the total space of this line bundle with a standard Kähler structure, whose restriction to the zero section is the spherical area form with total area 1. We denote the zero section as $C_{n}$.

Proposition 2.1. The topological group $\operatorname{Symp}_{c}(s L(n, 1))$ is weakly homotopy equivalent to $\operatorname{Symp}_{c}\left(\mathscr{O}(n) \backslash C_{n}\right)$.

Proof. Identifying $L(n, 1)$ as a circle bundle in $\mathscr{O}(n)$ with contact structure given by the connection 1-form, we get a canonical embedding:

$$
\mathscr{O}(n) \backslash C_{n} \hookrightarrow s L(n, 1),
$$

where the image is $\{(x, t) \in s L(n, 1): t<1\}$. Let $\operatorname{Symp}_{c}^{r}(s L(n, 1))$ be the subgroup of $\operatorname{Symp}_{c}(s L(n, 1))$ consisting of symplectomorphisms supported in $\{t<r\}$, then the embedding (2.2) induces an embedding of the corresponding groups of symplectomorphisms, where the image is exactly $\operatorname{Symp}_{c}^{1}(s L(n$, 1)). On the one hand, for $r>1$, using the inverse Liouville flow one sees that $\operatorname{Symp}_{c}^{r}(s L(n, 1))$ deformation retracts to $\operatorname{Symp}_{c}^{1}(s L(n, 1))$; on the other hand, $\operatorname{Symp}_{c}(s L(n, 1))$ is nothing but the direct limit of $\operatorname{Symp}_{c}^{r}(s L(n, 1))$ as $r \rightarrow \infty$. This concludes the proof.

\section{2. $\operatorname{Symp}_{c}\left(\mathscr{O}(n) \backslash C_{n}\right)$ as a loop space}

We will find the weak homotopy type of $\operatorname{Symp}_{c}\left(\mathscr{O}(n) \backslash C_{n}\right)$ in this section by showing it is weakly homotopy equivalent to a certain loop space. We start with some known results about $\operatorname{Symp}_{c}(\mathscr{O}(n))$.

Lemma 2.2. $\operatorname{Symp}_{c}(\mathscr{O}(n))$ is weakly contractible.

This result was shown in [5], Proposition 3.2. Coffey proceeded by compactifying $\mathscr{O}(n)$ by adding an infinity divisor to obtain the projectivization of $\mathscr{O}(n)$, which is the Hirzebruch surface $\mathbb{F}_{n}$. Symplectomorphisms of Hirzebruch surfaces are then studied using holomorphic curves. We note that this can also be deduced from Abreu and McDuff's results in [1]. 
Now, Coffey also showed that $\operatorname{Symp}_{c}(\mathscr{O}(n))$ acts transitively on the space $S\left(C_{n}\right)$ of unparametrized embedded symplectic spheres in $\mathscr{O}(n)$ which are homotopic to the zero section. We then have an action fibration

$$
\operatorname{Stab}_{c}\left(C_{n}\right) \rightarrow \operatorname{Symp}_{c}(\mathscr{O}(n)) \rightarrow S\left(C_{n}\right)
$$

where $\operatorname{Stab}_{c}\left(C_{n}\right)$ is the subgroup of $\operatorname{Symp}_{c}(\mathscr{O}(n))$ consisting of symplectomorphisms which preserve the zero section $C_{n}$.

Lemma 2.3 (Coffey [5]). The stabilizer $\operatorname{Stab}_{c}\left(C_{n}\right)$, is contractible.

Let $\mathcal{G}_{\omega}(\nu)$ be the symplectic gauge transformations of the normal bundle $\nu$ of $C_{n}$, that is, sections of $S p(\nu) \rightarrow C_{n}$, where $S p(\nu)$ are the fiberwise symplectic linear maps. Notice that $\mathcal{G}_{\omega}(\nu) \simeq \operatorname{Map}\left(C_{n}, S p(2)\right) \simeq S^{1}$ (see [7], [19]).

Let $\operatorname{Fix}_{c}\left(C_{n}\right)$ be the subgroup of $\operatorname{Stab}_{c}\left(C_{n}\right)$ consisting of symplectomorphisms which fix the zero section $C_{n}$ pointwise. We will use the following lemma from time to time.

Lemma 2.4. The homomorphism $\operatorname{Fix}_{c}\left(C_{n}\right) \rightarrow \mathcal{G}_{\omega}(\nu)$ given by taking derivatives along $C_{n}$ is surjective.

Proof. Let $g \in \mathcal{G}_{\omega}(\nu)$. Then each $g(z)$ for $z \in S^{2}$ is a symplectic transformation of the normal fiber $\nu_{z}$ over $z$. Any such linear symplectic map is the time 1 Hamiltonian flow $\phi_{1}$ of a unique quadratic form $Q(z)$ on $\nu_{z}$.

Consider the Hamiltonian function $H(z, v)=\chi(|v|) Q(z) v$ on $\mathscr{O}(n)$, where $\chi$ is a bump function equal to 1 near 0 and 0 when $|v| \geq 1$. As $d H=0$ along $C_{n}$ the resulting Hamiltonian flow $\psi_{t}$ lies in $\operatorname{Fix}_{c}\left(C_{n}\right)$. We will check that the corresponding gauge action at time 1 is precisely $g$.

For this, let $Y \in \nu_{z} \cong T_{0} \nu_{z} \subset T_{z} \mathscr{O}(n)$. Then we claim that $d \psi_{t}(Y)=$ $\phi_{t}(Y)$, where in the second term $Y$ is considered as a point in $\nu_{z}$ and $\phi_{t}$ is the Hamiltonian flow of $Q: \nu_{z} \rightarrow \mathbb{R}$. The vector $Y$ can be extended to a Hamiltonian vector field on $\mathscr{O}(n)$ generated by a function $L$ which is linear on $\nu_{z}$. Let $X_{H}$ be the Hamiltonian vector field generated by $H$. Then

$$
\mathcal{L}_{X_{H}} Y=\left[X_{H}, Y\right]=X_{\{H, L\}}=X_{d H(Y)}
$$

using the same notation throughout for Hamiltonian vector fields. Evaluating at $z$, our Lie derivative is tangent to the fiber $\nu_{z}$, and restricting to this fiber the function $d H(Y)=d Q(z)(Y)$ is linear and dual under the symplectic form to $X_{Q}(Y)$. In other words, $\mathcal{L}_{X_{H}} Y(z)=X_{Q}(Y)$, identifying two vectors in $\nu_{z}$. This is equivalent to our claim and so the proof is complete. 
Let $\operatorname{Fix}_{c}^{\text {id }}\left(C_{n}\right)$ denote the subgroup of $\operatorname{Fix}_{c}\left(C_{n}\right)$ consisting of diffeomorphisms whose derivatives act trivially on the normal bundle $\nu$ of the zero section. A simple application of Moser's argument shows that $\operatorname{Fix}_{c}^{\text {id }}\left(C_{n}\right)$ is homotopy equivalent to $\operatorname{Symp}_{c}\left(\mathscr{O}(n) \backslash C_{n}\right)$, and we will freely switch between these two groups without explicitly mentioning it below.

Let us write $\operatorname{Aut}_{\omega}(\nu)$ for the group of automorphisms of the normal bundle $\nu$ of the zero section $C_{n}$ which are symplectic linear on the fibers and preserve the symplectic form along the zero section. The group $\operatorname{Stab}_{c}\left(C_{n}\right)$ acts on $\operatorname{Aut}_{\omega}(\nu)$ via its derivative along the zero section. Clearly $\operatorname{Stab}_{c}\left(C_{n}\right)$ acts transitively on $C_{n}$ and so by Lemma 2.4 the action on $\operatorname{Aut}_{\omega}(\nu)$ is also transitive. Hence we have the fibration

$$
\operatorname{Fix}_{c}^{\text {id }}\left(C_{n}\right) \longleftrightarrow \operatorname{Stab}_{c}\left(C_{n}\right) \longrightarrow \operatorname{Aut}_{\omega}(\nu)
$$

which by Lemma 2.3 yields a weak homotopy equivalence (cf. Proposition $4.66[9])$

$$
\operatorname{Fix}_{c}^{\text {id }}\left(C_{n}\right) \simeq \mathscr{L} \operatorname{Aut}_{\omega}(\nu)
$$

where $\mathscr{L} \operatorname{Aut}_{\omega}(\nu)$ is the space of based loops of $\operatorname{Aut}_{\omega}(\nu)$. Therefore, the following proposition will imply the first part of Theorem 1.1:

Proposition 2.5. The group $\operatorname{Aut}_{\omega}(\nu)$ is homotopy equivalent to the Kähler isometry group $K_{n}$ of the Hirzebruch surface $\mathbb{F}_{n}$. In particular,

$$
\operatorname{Aut}_{\omega}(\nu) \simeq K_{n} \simeq U(2) / \mathbb{Z}_{n} \simeq \begin{cases}S O(3) \times S^{1} & \text { if } n \text { is even, } n \neq 0 \\ U(2) & \text { if } n \text { is odd }\end{cases}
$$

so that $\mathscr{L} \operatorname{Aut}_{\omega}(\nu)$ has countably many components, where each component is homotopy equivalent to $\mathscr{L} S U(2)$, that is, to the identity component of $\mathscr{L} S O(3)$.

Proof. First notice that $\operatorname{Aut}_{\omega}(\nu)$ acts transitively on the symplectic reparametrization group of the zero section, or equivalently, the symplectomorphism group of $\mathbb{C} P^{1}$. We thus have an action fibration

$$
\mathcal{G}_{\omega}(\nu) \longleftrightarrow \operatorname{Aut}_{\omega}(\nu) \longrightarrow \operatorname{Symp}\left(\mathbb{C} P^{1}\right)
$$

whose fiber is the subgroup which fixes $\mathbb{C} P^{1}$ pointwise and thus is simply the gauge group $\mathcal{G}_{\omega}(\nu)$.

Recall that the Hirzebruch surface $\mathbb{F}_{n}$ is the projectivation $\mathbb{P}(\mathscr{O}(n) \oplus \mathbb{C})$. Under the action of its Kähler isometry group $K_{n} \simeq U(2) / \mathbb{Z}_{n}$, the complex 
surface $\mathbb{F}_{n}$ can be partitioned into three invariant parts: the zero section $C_{n}$, the section at infinity $C_{n}^{\infty}$ and their open complement $\mathbb{F}_{n} \backslash\left\{C_{n} \cup C_{n}^{\infty}\right\}$, see Appendix B in [2]. Since the $K_{n}$ action preserves the ruling $\mathbb{F}_{n} \rightarrow \mathbb{C} P^{1}$, every element in $K_{n}$ acts as an isometry of $\mathbb{C} P^{1}$ and $K_{n}$ acts faithfully on the normal bundle $\nu$ on $C_{n}$ via derivatives. We thus get a commutative diagram of fibrations

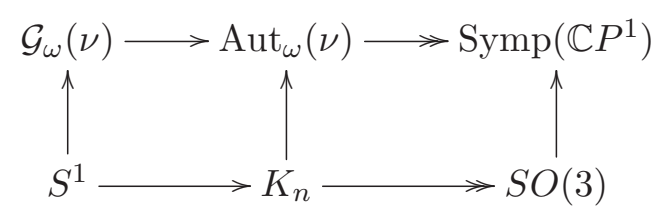

in which the first and third vertical inclusions are homotopy equivalences. It follows that the middle inclusion is a weak homotopy equivalence. Since all spaces involved are homotopy equivalent to CW-complexes, this weak equivalence is a genuine homotopy equivalence. The second part of the statement now follows from substituting $M=S O(3)$ and $N=K_{n}$ in the following simple lemma:

Lemma 2.6. Let $M$ be a $C W$-complex with $\pi_{2}(M)=0$ and $\pi_{1}(M)$ at most countable. Suppose $N$ is an $S^{1}$-bundle over $M$. Then $\mathscr{L}(N)$ has countably many components and we have a weak homotopy equivalence between identity components $\mathscr{L}^{0}(N) \simeq \mathscr{L}^{0}(M)$.

Proof of the lemma. This fact is an elementary consequence of the usual "path-loop" construction. Fix a base point on $N$ and let $P(N) \simeq *$, be the corresponding based path space. The fibration map $\pi: N \rightarrow M$ induces the commutative diagram:

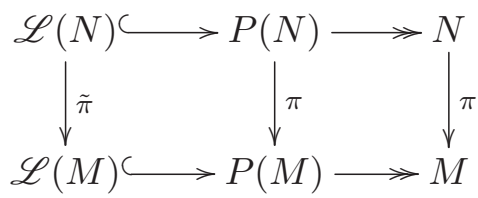

By assumption, the projections $\pi_{*}: \pi_{k}(N) \rightarrow \pi_{k}(M)$, are isomorphisms for $k \geq 2$, and the circle fiber and its multiples are non-zero in $\pi_{1}(N)$. From the commutative diagram of the long exact sequence of homotopy groups induced by (2.5), we deduce that:

$$
\tilde{\pi}_{*}: \pi_{k}(\mathscr{L}(N)) \stackrel{\cong}{\longrightarrow} \pi_{k}(\mathscr{L}(M)), \quad \text { when } k \geq 1
$$


Moreover, we have noticed that $\pi_{1}(N)$ is the central extension of $\mathbb{Z}$ and $\pi_{1}(M)$, hence the lemma follows.

This concludes the proof of Proposition 2.5

\subsection{The loop group of the contact isometries of $L(n, 1)$}

In this section, we prove the second part of Theorem 1.1 by showing that a natural map is a weak homotopy equivalence. Recall that in Section 2.2 we have shown that there exists a weak homotopy equivalence between our symplectomorphism group $\operatorname{Symp}_{c}(s L(n, 1))$ and the loop group $\mathscr{L} \operatorname{Aut}_{\omega}(\nu)$. We will see in a moment that the contact isometries $\mathcal{C} \mathrm{Iso}_{n}$ are weakly homotopic to $\operatorname{Aut}_{\omega}(\nu)$ and therefore we already have a weak homotopy equivalence between $\mathscr{L}\left(\mathcal{C} \operatorname{Iso}_{n}\right)$ and $\operatorname{Symp}_{c}(s L(n, 1))$. The main goal of this section is to show that this weak homotopy equivalence is induced by a natural inclusion map.

Unlike the usual notion of contactomorphism which preserves only the contact structures, we need to consider the automorphisms of $L(n, 1)$ called contact isometries. These are diffeomorphisms which preserve the contact form $\lambda_{n}$ and the round metric induced from the round metric on $S^{3}$ under projection. We denote the group of contact isometries of the lens spaces of $L(n, 1)$ as $\mathcal{C} \mathrm{Iso}_{n}$. It acts on $L(n, 1)$ in such a way that the Reeb orbits are preserved. Therefore, if we think of $L(n, 1)$ as a unit circle bundle in $\mathscr{O}(n)$ with the Reeb orbits as the circle fibers, there is an induced isometric action of $\mathcal{C}$ Iso $_{n}$ on the base $\mathbb{C} P^{1}$ endowed with the standard round metric. Also, since the action on the fibers is linear, there is a natural inclusion $\mathcal{C} \mathrm{Iso}_{n} \hookrightarrow \operatorname{Aut}_{\omega}(\nu)$. Therefore, along with (2.4), one obtains the following diagram of fibrations :

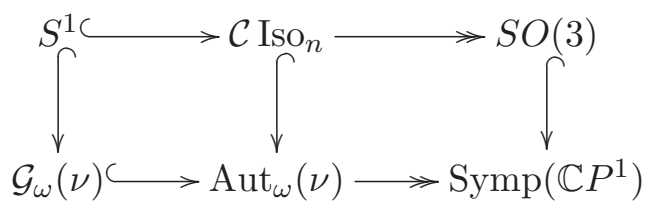

Notice that we have weak homotopy equivalences in both the base and fiber. Therefore, the natural inclusion of $\mathcal{C} \operatorname{Iso}_{n}$ into $\operatorname{Aut}_{\omega}(\nu)$ is in fact a (weak) homotopy equivalence.

We now want to describe a natural map from $\mathscr{L}\left(\mathcal{C} \operatorname{Iso}_{n}\right)$ to $\operatorname{Symp}_{c}(\mathscr{O}(n) \backslash$ $C_{n}$ ) (or equivalently $\operatorname{Fix}_{c}^{\text {id }}\left(C_{n}\right)$, see Section 2.2 ) which induces a weak homotopy equivalence. Given Proposition 2.1 this will imply the remainder of 
Theorem 1.1. To this end, consider the smooth path space

$$
P\left(\mathcal{C} \mathrm{Iso}_{n}\right)=\left\{\phi:(-\infty,+\infty) \rightarrow \mathcal{C} \mathrm{Iso}_{n}: \phi(t)=i d, t \leq 0, \phi(t)=\phi(1), t \geq 1\right\} .
$$

This is just the usual based path space when restricted to $t \in[0,1]$, thus it is a contractible space. Given $\phi \in P\left(\mathcal{C} \mathrm{Iso}_{n}\right)$ one can define the following diffeomorphism of $s L(n, 1)$ :

$$
\begin{aligned}
\phi^{\prime}: L(n, 1) \times \mathbb{R} & \longrightarrow L(n, 1) \times \mathbb{R} \\
(x, t) & \longmapsto(\phi(t) x, t)
\end{aligned}
$$

By definition, $\left.\phi^{\prime}\right|_{t \leq 0}=i d$, and $\left.\phi^{\prime}\right|_{1 \leq t \leq 2}$ is a symplectomorphism induced by a contact isometry multiplied by identity in the $\mathbb{R}$-direction. However $\phi^{\prime}$ fails to be a symplectomorphism in general. Let $\omega_{0}=d\left(e^{t} \lambda_{n}\right)$, the canonical symplectic form on $s L(n, 1)$, and $\omega_{1}=\phi^{*} \omega_{0}$. Then nevertheless we claim that the exact forms $\omega_{u}=(1-u) \omega_{0}+u \omega_{1}$ are symplectic for all $0 \leq u \leq 1$.

Proof of claim. To see this, arguing by contradiction, note that if an $\omega_{u}$ fails to be symplectic then it has a kernel of dimension at least 2, which must intersect the tangent space to some level $L(n, 1) \times\{t\}$ nontrivially. As our $\phi(t)$ are contact isometries this kernel must be the kernel of $d \lambda_{n}$, namely the Reeb direction. But as the Reeb direction is preserved and $\phi_{*}^{\prime}\left(\frac{\partial}{\partial t}\right)$ always has a positive $\frac{\partial}{\partial t}$ component, the Reeb vector pairs nontrivially with $\frac{\partial}{\partial t}$ under all $\omega_{u}$.

Given our claim, we can apply Moser's method, see [18], that is, we compose $\phi^{\prime}$ with the flow of the time-dependent vector field defined by $\left.X_{u}\right\rfloor \omega_{u}=\phi^{\prime *}\left(e^{t} \lambda_{n}\right)-e^{t} \lambda_{n}$. The composition is a symplectomorphism $\tilde{\phi}$ of $s L(n, 1)$ supported in $\{t \geq 0\}$. On $\{t \geq 1\}$ we have $\phi^{\prime *}\left(e^{t} \lambda_{n}\right)=e^{t} \lambda_{n}$ and so on this region we have $\left.\tilde{\phi}\right|_{t \geq 1}=\left.\phi^{\prime}\right|_{t \geq 1}$.

Next, as $\phi^{\prime}$ is translation invariant on $\{t \geq 1\}$ we can perform a symplectic cut at the level of $\{t=2\}$. Recall from Section 2.1 that, up to a scaling, we may identify symplectically $\mathscr{O}(n) \backslash C_{n}$ with $s L(n, 1)_{t<2}$. Hence $\tilde{\phi}$ descends to a compactly supported symplectomorphism of $\mathscr{O}(n)$ preserving the zero-section, that is, we have a map $P\left(\mathcal{C} \operatorname{Iso}_{n}\right) \rightarrow \operatorname{Stab}_{c}\left(C_{n}\right), \phi \mapsto \tilde{\phi}$.

Claim: The following diagram of fibrations is commutative and all maps are continuous. The rightmost vertical arrow is a homotopy equivalence: 


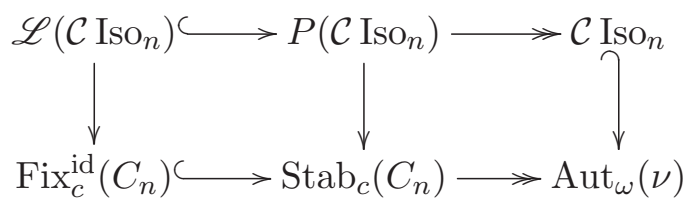

Proof of claim. The second arrow of the first row is simply the restriction of an element $\phi$ to $\phi(2)$. The continuity of the vertical maps follows from the continuous dependence of solutions of an ODE on initial conditions when applying Moser's method. The rightmost vertical arrow is the one induced from (2.7) and is thus a homotopy equivalence. The commutativity of the diagram (2.10) is straightforward from definitions.

Now the middle vertical arrow is a homotopy equivalence due to the contractibility of both spaces, see Lemma 2.3, and the rightmost arrow is also a weak homotopy equivalence from the argument at the start of this subsection. Therefore, the leftmost vertical arrow is a homotopy equivalence as well, and provides the desired mapping. Hence, the second part of Theorem 1.1 follows.

\section{Space of symplectic embeddings of orbifold balls}

In this section, we study the space of symplectic embeddings of balls with a single conical singularity at the origin. We first briefly recall the two different notions of maps between orbifolds that we use and the related definition of orbifold embeddings. A comprehensive discussion of orbifold structures and of orbifold maps can be found in [4].

Given an orbifold $\mathcal{A}$, we write $|\mathcal{A}|$ for its underlying topological space. An unreduced orbifold map $(f,\{\hat{f}\})$ between two orbifolds $\mathcal{A}$ and $\mathcal{B}$ consists of the following data:

1) a continuous map $f:|\mathcal{A}| \rightarrow|\mathcal{B}|$ of the underlying topological spaces;

2) for all $x \in|\mathcal{A}|$, the choice of a germ $\hat{f}_{x}$ of local lift of $f$ to uniformizing charts $U$ and $V$ centered at $x$ and $f(x)$.

A reduced orbifold map is a continuous map $f:|\mathcal{A}| \rightarrow|\mathcal{B}|$ of the underlying topological spaces such that smooth lifts exist at every point. The set of smooth unreduced orbifold maps between $\mathcal{A}$ and $\mathcal{B}$ will be denoted by $C_{\text {orb }}^{\infty}(\mathcal{A}, \mathcal{B})$, while we will write $C_{\text {red }}^{\infty}(\mathcal{A}, \mathcal{B})$ for the set of smooth reduced orbifold maps. Smooth unreduced or reduced diffeomorphisms are defined 
accordingly by requiring $f$ to be a homeomorphism and all lifts to be smooth local diffeomorphisms. The sets of all unreduced or reduced diffeomorphisms of an orbifold $\mathcal{A}$ can be naturally endowed with a $C^{\infty}$ topology that make them Fréchet Lie groups. The short exact sequence

$$
1 \rightarrow \Gamma_{\text {id }} \rightarrow \operatorname{Diff}_{\text {orb }}(\mathcal{A}) \rightarrow \operatorname{Diff}_{\text {red }}(\mathcal{A}) \rightarrow 1
$$

is then a principal bundle whose fiber $\Gamma_{\text {id }}$ is the (discrete) group of all lifts of the identity map.

A smooth (unreduced, resp. reduced) embedding $f: \mathcal{A} \rightarrow \mathcal{B}$ is a smooth (unreduced, resp. reduced) orbifold map which is a diffeomorphism onto its image and which covers a topological embedding $f:|\mathcal{A}| \rightarrow|\mathcal{B}|$. We will denote by $\operatorname{Emb}_{\text {orb }}(\mathcal{A}, \mathcal{B})$ and $\operatorname{Emb}_{\text {red }}(\mathcal{A}, \mathcal{B})$ the corresponding embedding spaces.

If all the uniformizing charts are symplectic, and if all the local group actions preserve the symplectic forms, the orbifold atlas is said to be symplectic. Orbifold symplectic maps are then defined in the obvious way. In particular, since the open set of regular points $\mathcal{A}_{\text {reg }}$ becomes an open symplectic manifold, orbifold symplectomorphisms restrict to genuine smooth symplectomorphisms of $\mathcal{A}_{\text {reg }}$.

Let us write $B_{n}(\epsilon)$ for a symplectic ball of size $\epsilon$ with a single conical singularity of order $n \geq 1$ at the origin, that is,

$$
B_{n}(\epsilon):=B^{4}(n \epsilon) / \mathbb{Z}_{n}
$$

where $B^{4}(r)$ stands for the standard ball of radius $\sqrt{r / \pi}$ in $\mathbb{R}^{4}$ and $\mathbb{Z}_{n}$ acts as in (2.1). In this paper, we are only interested in the simplest possible embedding spaces between symplectic orbifolds, namely $\operatorname{Emb}_{r e d}\left(B_{n}(\epsilon), B_{n}(1)\right)$. In that case, it is easy to see that $\operatorname{Emb}_{\text {orb }}\left(B_{n}(\epsilon), B_{n}(1)\right)$ consists of smooth symplectic embeddings $f: B^{4}(n \epsilon) \rightarrow B^{4}(n)$ of standard smooth balls that are equivariant with respect to the standard $\mathbb{Z}_{n}$ action, and that

$$
\operatorname{Emb}_{r e d}\left(B_{n}(\epsilon), B_{n}(1)\right)=\operatorname{Emb}_{\text {orb }}\left(B_{n}(n \epsilon), B_{n}(n)\right) / \mathbb{Z}_{n}
$$

This follows from the fact that any local smooth lift at the conical point extends uniquely to the whole ball, see [4]. Since the space of smooth symplectic embeddings retracts onto $U(2)$, and since the $\mathbb{Z}_{n}$ action belongs to the center of $U(2)$, one can show that the space of $\mathbb{Z}_{n}$-equivariant embeddings of smooth balls is itself homotopy equivalent to $U(2)$, see [22]. Therefore, we have the following results: 
Proposition 3.1. The space of reduced symplectic embeddings $\operatorname{Emb}_{\text {red }}\left(B_{n}(\epsilon)\right.$, $\left.B_{n}(1)\right)$ is homotopy equivalent to

$$
K_{n}:=U(2) / \mathbb{Z}_{n} \simeq \begin{cases}S O(3) \times S^{1} & \text { if } n \text { is even, } n \neq 0 \\ U(2) & \text { if } n \text { is odd }\end{cases}
$$

Just as in the smooth case, one can show that the group of compactly supported and reduced symplectomorphisms of the open orbifold ball $B_{n}(1)$ acts transitively on $\operatorname{Emb}_{\text {red }}\left(B_{n}(\epsilon), B_{n}(1)\right)$, see [22]. We get an action fibration

$$
\operatorname{Stab}_{c, r e d}(\iota) \rightarrow \operatorname{Symp}_{c, r e d}\left(B_{n}(1)\right) \rightarrow \operatorname{Emb}_{\text {red }}\left(B_{n}(\epsilon), B_{n}(1)\right)
$$

where $\iota: B_{n}(\epsilon) \rightarrow B_{n}(1)$ is the inclusion, and where $\operatorname{Stab}_{c, r e d}(\iota)$ is the subgroup made of those reduced symplectomorphisms that are the identity on the image $\iota\left(B_{n}(\epsilon)\right)$. This subgroup is homotopy equivalent to the group of reduced diffeomorphisms that are the identity near the image $\iota\left(B_{n}(\epsilon)\right)$. Performing a symplectic blow-up of the ball $\iota\left(B_{n}(\epsilon)\right)$, those symplectomorphisms lift to symplectomorphisms of the Hirzebruch surface $\mathbb{F}_{n}$ that are the identity near the zero section and near the section at infinity. This last group is itself homotopy equivalent to $\operatorname{Symp}_{c}(s L(n, 1))$. Hence, we get a homotopy fibration

$$
\operatorname{Symp}_{c}(s L(n, 1)) \rightarrow \operatorname{Symp}_{c, r e d}\left(B_{n}(1)\right) \rightarrow \operatorname{Emb}_{r e d}\left(B_{n}(\epsilon), B_{n}(1)\right)
$$

which shows that the homotopy equivalence $\operatorname{Symp}_{c}(s L(n, 1)) \simeq \mathscr{L} K_{n}$, together with Proposition 3.1, imply the following mild generalization of a fundamental result due to Gromov:

Proposition 3.2. The group $\operatorname{Symp}_{c, \text { red }}\left(B_{n}(1)\right)$ of reduced, compactly supported symplectomorphisms of an open ball of size 1 with a single conical singularity of order $n$ at the origin is contractible.

This completes the proof of Theorem 1.2.

\section{Space of Lagrangian $\mathbb{R} \boldsymbol{P}^{2}$ in $T^{*} \mathbb{R} \boldsymbol{P}^{2}$}

In this section we prove Theorem 1.3. Let the space of Lagrangian $\mathbb{R} P^{2}$ in $T^{*} \mathbb{R} P^{2}$ be denoted by $\mathcal{L}$. The group of compactly supported Hamiltonian symplectomorphisms of $T^{*} \mathbb{R} P^{2}$ acts transitively on $\mathcal{L}$, see [12], and our point 
of departure is the corresponding action fibration

$$
\operatorname{Stab}_{c}(\mathbf{0}) \longleftrightarrow \operatorname{Symp}_{c}\left(T^{*} \mathbb{R} P^{2}\right) \longrightarrow \mathcal{L}
$$

where $\operatorname{Stab}_{c}(\mathbf{0})$ is the subgroup of $\operatorname{Symp}_{c}\left(T^{*} \mathbb{R} P^{2}\right)$ which preserves the zero section $\mathbf{0 .}$

Notice that for any Lie group $G, \pi_{0}(G)$ inherits a natural group structure from $G$. It is proved in [7] that:

Theorem 4.1. $\operatorname{Symp}_{c}\left(T^{*} \mathbb{R} P^{2}\right)$ is weakly homotopic to $\mathbb{Z}$. Moreover, the generator of $\pi_{0}\left(\operatorname{Symp}_{c}\left(T^{*} \mathbb{R} P^{2}\right)\right)$ as a group is the generalized Dehn twist in $T^{*} \mathbb{R} P^{2}$.

We will also make use of the following fact, which may be well-known but for which the authors unfortunately know of no reference:

Lemma 4.2. Let $H \longleftrightarrow G \longrightarrow B$ be a homotopy fibration where $H \triangleleft G$ are groups. Then the following two maps in the induced long exact sequence are both group homomorphisms:

$$
\pi_{1}(B) \stackrel{i}{\longrightarrow} \pi_{0}(H) \stackrel{j}{\longrightarrow} \pi_{0}(G)
$$

Proof. Let $x_{0} \in B$ be the image of $i d \in G$. Given a loop $\alpha:[0,1] \rightarrow B$, $\alpha(0)=\alpha(1)=x_{0}$, let $\bar{\alpha}$ be the lift of $\alpha$ and $i(\alpha)$ be the connected component of $H$ where $\bar{\alpha}(1)$ lies. Consider another loop $\beta$ : $[0,1] \rightarrow B, \beta(0)=\beta(1)=x_{0}$, then the lift of concatenation $\alpha \# \beta$ can be chosen to be

$$
\overline{\alpha \# \beta}(t)=\left\{\begin{aligned}
\bar{\alpha}(2 t), & t \leq \frac{1}{2} \\
\bar{\alpha}(1) \cdot \bar{\beta}(2 t-1), & t>\frac{1}{2}
\end{aligned}\right.
$$

Therefore, $i(\alpha \# \beta)=\overline{\alpha \# \beta}(1)=\bar{\alpha}(1) \bar{\beta}(1)$, verifying the claim for the map $i$. The fact that $j$ is a homomorphism is trivial because the inclusion $H \hookrightarrow G$ is a homomorphism.

To compute the homotopy type of $\operatorname{Stab}_{c}(\mathbf{0})$ we need to consider the diffeomorphism group of $\mathbb{R} P^{2}$. We have the following result, of which the proof is postponed to the appendix:

Proposition 4.3. The diffeomorphism group of $\mathbb{R} P^{2}$ is weakly homotopic to $S O(3)$. Moreover, the standard inclusion is a weak homotopy equivalence. 
With this understood, we define $\operatorname{Fix}_{c}(\mathbf{0})$ to be the subgroup of compactly supported symplectomorphisms of $T^{*} \mathbb{R} P^{2}$ which fix the zero section pointwise. We obtain a further action fibration:

$$
\operatorname{Fix}_{c}(\mathbf{0}) \longleftrightarrow \operatorname{Stab}_{c}(\mathbf{0}) \longrightarrow \operatorname{Diff}\left(\mathbb{R} P^{2}\right)
$$

We may also consider the following object: given the standard round metric $g_{0}$ on $\mathbb{R} P^{2}$, let $\operatorname{Stab}_{c}^{\text {Iso }}(\mathbf{0})$ be the symplectomorphisms which are compactly supported and induce an isometry on the zero section.

Now we have the following commutative diagram of fibrations:

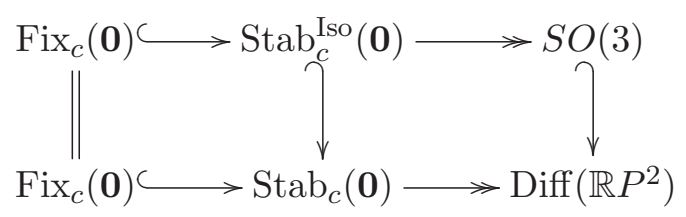

From Proposition 4.3, we observe that the vertical arrows on the two sides are weak homotopy equivalences, so the middle one is also a weak homotopy equivalence. Note also that the inverse Liouville flow contracts $T^{*} \mathbb{R} P^{2}$ to the zero section. Now, taking into consideration the bundle metric on $T^{*} \mathbb{R} P^{2}$ induced by $g_{0}$, we may talk about the length of cotangent vectors. By the same direct limit and Liouville flow argument as in Proposition 2.1, when studying compactly supported symplectomorphism groups of $T^{*} \mathbb{R} P^{2}$ we may restrict our attention to the symplectomorphisms supported in $T_{r}^{*} \mathbb{R} P^{2}$, which consists of cotangent vectors with length $\leq r$ for some $r>0$. We will assume that $r=\frac{1}{2}$ below.

Most of the remainder of this section will be occupied with proving the following.

Lemma 4.4. (i) $\operatorname{Stab}_{c}^{\text {Iso }}(\mathbf{0})$ is weakly homotopy equivalent to $\mathbb{Z}$;

(ii) $\pi_{0}\left(\operatorname{Stab}_{c}^{\text {Iso }}(\mathbf{0})\right)$ is isomorphic to $\mathbb{Z}$ as a group.

Remark 4.5. It is very tempting to conclude $(i)$ directly from the results in the previous sections by setting $n=4$, see the first paragraph of the proof. However, the connecting map in (4.2) seems then difficult to understand directly. That is why we use a slightly different argument below.

Proof of Lemma 4.4. We first notice the following fact: a symplectomorphism which fixes a smooth Lagrangian pointwise also fixes the framing of the Lagrangian. This follows from the corresponding linear statement 
that, a symplectomorphism of $T^{*} M$ which is linear on the fibers is indeed a cotangent map of a diffeomorphism on the base. This is also used in [5], proof of Theorem 1.3. It follows that the $\operatorname{subgroup~of~} \operatorname{Stab}_{c}^{\text {Iso }}(\mathbf{0})$ consisting of maps which act on a neighborhood of $\mathbb{R} P^{2}$ by the cotangent map of an isometry of $\mathbb{R} P^{2}$ is weakly homotopy equivalent to $\operatorname{Stab}_{c}^{\text {Iso }}(\mathbf{0})$. Therefore we are able to consider this subgroup instead of $\operatorname{Stab}_{c}^{\text {Iso }}(\mathbf{0})$, and use the same notation to denote it throughout the rest of the proof.

Our approach is to convert the study of maps supported near a Lagrangian to maps supported in a neighborhood of a positive symplectic curve, where symplectic techniques are more convenient. Given $\psi \in$ $\operatorname{Stab}_{c}^{\text {Iso }}(\mathbf{0})$, denoting the cotangent map of $\left.\psi\right|_{\mathbb{R} P^{2}}$ as $c_{\psi}$, we may consider the symplectomorphism $\tilde{\psi}:=c_{\psi}^{-1} \circ \psi$ on $T^{*} \mathbb{R} P^{2}$. The map $\tilde{\psi}$ is not compactly supported in $T^{*} \mathbb{R} P^{2}$, but it fixes $\mathbb{R} P^{2}$ pointwise and thus (by our assumption that the maps are cotangents near $\mathbb{R} P^{2}$ ) also a neighborhood. Since $\psi$ is the identity outside $r \leq 1$, we have $\widetilde{\psi}=c_{\psi}^{-1}$ outside $r \leq 1$. Also, since $\psi$ preserves the round metric on $\mathbb{R} P^{2}, c_{\psi}$ preserves the Reeb vector field on the level sets of $T^{*} \mathbb{R} P^{2}$. Therefore, by a symplectic cut on the level set $r=1$, one obtains a symplectomorphism $\psi^{\prime}$ of $T^{*} \mathbb{R} P^{2}$ cut along the level $r=1$. This symplectic manifold is just $\mathbb{C} P^{2}$ with the standard symplectic form $\omega_{F S}$, see [3] and [14]. From the construction, $\psi^{\prime}$ preserves the symplectic reduction of the boundary, a symplectic $(+4)$-sphere which we identify with the quadratic sphere $C_{4}=\left\{[x, y, z] \in \mathbb{C} P^{2}: x^{2}+y^{2}+z^{2}=0\right\}$, and it fixes a neighborhood of the standard Lagrangian $\mathbb{R} P^{2}=\operatorname{Re}\left(\mathbb{C} P^{2}\right)$. Removing the Lagrangian $\mathbb{R} P^{2}$, one sees that $\psi^{\prime}$ descends to a compactly supported symplectomorphism of $\mathscr{O}(4)$, which is denoted by $\bar{\psi}$.

Definition 4.6. The subgroup $H \subset \operatorname{Symp}_{c}(\mathscr{O}(4))$ is the image of the bar assignment $\psi \mapsto \bar{\psi}, \operatorname{Stab}_{c}^{\text {Iso }}(\mathbf{0}) \rightarrow \operatorname{Symp}_{c}(\mathscr{O}(4))$.

From the construction, $H$ is clearly isomorphic to $\operatorname{Stab}_{c}^{\text {Iso }}(\mathbf{0})$ as a group, thus also homeomorphic. In the rest of the proof we investigate the homotopy type of $H$.

Warning: Although $H$ has the property that every element acts fiberwise linearly in a neighborhood of the quadric surface, it is not the whole subgroup of $\operatorname{Symp}_{c}(\mathscr{O}(4))$ with this property. This is because the action of an element $h \in H$ on a neighborhood of the quadric surface is completely determined by the action on the surface itself. Therefore, heuristically speaking, the gap between $H$ and the subgroup of fiberwise linear action is the whole gauge transformation on the normal bundle, as we will see momentarily. For interested readers we have included an explicit description of $H$, pointed 
out to us by Yi Liu, at the end of the proof of Lemma 4.4 (Remark 4.11). However this will not be needed for our proof.

Let $U$ be a neighborhood of the zero section in $\mathscr{O}(4)$, then let $\left.H\right|_{U} \subset$ $\operatorname{Symp}(U)$ be the symplectomorphisms of $U$ given by restricting elements of $H$. Another subgroup of $\operatorname{Symp}(U)$ corresponds to the restriction of the cotangent maps of isometries of $\mathbb{R} P^{2}$. As the isometry group is $S O(3)$ we can unambiguously denote this subgroup by $S O(3)$. Remembering that points of the zero section in $\mathscr{O}(4)$ correspond to the lifts of a geodesic we see that $S O(3)$ acts transitively on the zero section. Now, for $U$ sufficiently small, for example the region $\left\{r \geq \frac{1}{2}\right\}$, all elements of $\left.H\right|_{U}$ are of the form $c_{\psi}^{-1}$, that is, they are elements of $S O(3)$. On the other hand, as any isometry of $\mathbb{R} P^{2}$ is the restriction of a $\psi \in \operatorname{Stab}_{c}^{\text {Iso }}(\mathbf{0})$, all elements of $S O(3)$ arise this way. Hence we have the following.

Lemma 4.7. Let $U$ be a sufficiently small neighborhood of the zero section in $\mathscr{O}(4)$, then $\left.H\right|_{U}=S O(3)$, and it acts transitively on the zero section.

Given the round metric $g$ on the zero section $C_{4}$ of $\mathscr{O}(4)$, we consider the subgroups

$$
\begin{aligned}
& \operatorname{Stab}_{c}\left(C_{4}\right)=\left\{\psi \in \operatorname{Symp}_{c}(\mathscr{O}(4)): \psi \text { preserves the zero section } C_{4}\right\} \\
& \operatorname{Stab}_{c}^{\text {Iso }}\left(C_{4}\right)=\left\{\psi \in \operatorname{Stab}_{c}\left(C_{4}\right): \psi\right. \text { restricted to the zero section } \\
&\text { is an isometry with respect to the metric } g\}
\end{aligned}
$$

$$
\operatorname{Fix}_{c}\left(C_{4}\right)=\left\{\psi \in \operatorname{Stab}_{c}^{\text {Iso }}\left(C_{4}\right):\left.\psi\right|_{C_{4}}=\mathrm{id}\right\}
$$

Lemma 4.8. $\operatorname{Stab}_{c}^{\mathrm{Iso}}\left(C_{4}\right)$ is weakly contractible.

Proof. We again have a commutative diagram of fibrations:

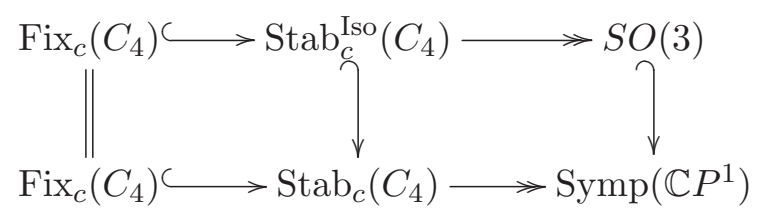

Using the fact that the embedding of $S O(3)$ into $\operatorname{Symp}\left(\mathbb{C} P^{1}\right)$ is a weak homotopy equivalence and Lemma 2.3, we deduce that $\operatorname{Stab}_{c}^{\text {Iso }}\left(C_{4}\right)$ is also weakly contractible. 
Let us construct a group homomorphism from $\operatorname{Stab}_{c}^{\text {Iso }}\left(C_{4}\right)$ to the gauge group:

$$
\phi: \operatorname{Stab}_{c}^{\text {Iso }}\left(C_{4}\right) \longrightarrow \operatorname{Map}\left(S^{2}, S p(2)\right) \simeq S^{1}
$$

To define $\phi$, let $t \in \operatorname{Stab}_{c}^{\text {Iso }}\left(C_{4}\right)$. Then $\left.t\right|_{C_{4}}$ acts on the zero section $C_{4}$ isometrically. Thinking of $S O(3)$ as a subgroup of $\operatorname{Symp}(\mathscr{O}(4)$ as above, there exists a unique element $u \in S O(3)$ such that $\left.u\right|_{C_{4}}=\left.t\right|_{C_{4}}$. Now we define $\phi(t)$ to be the gauge of $t \cdot u^{-1}$.

Lemma 2.4 implies that $\operatorname{Fix}_{c}\left(C_{4}\right) \subset \operatorname{Stab}_{c}^{\text {Iso }}\left(C_{4}\right)$ maps surjectively to the gauge group and hence we have the following.

Lemma 4.9. $\phi$ is a surjective homomorphism.

Now we return to the subgroup $H \subset \operatorname{Stab}_{c}^{\text {Iso }}\left(C_{4}\right)$.

Lemma 4.10. $\operatorname{ker}(\phi)$ is weakly homotopic to $H$.

Proof. From Lemma 4.7 and the definition of $\phi$, we have that $H \subset \operatorname{ker}(\phi)$. To see that this inclusion is a homotopy equivalence, take $t \in \operatorname{ker}(\phi)$. Again by Lemma 4.7 there exists a $u \in H$, such that $t \cdot u^{-1}$ acts trivially on the normal bundle of $C_{4}$. Therefore, up to homotopy, $\operatorname{ker}(\phi)$ consists of $t$ for which there is a $u \in H$ such that $t \cdot u^{-1}$ acts trivially on a neighborhood of $C_{4}$. Such $t \cdot u^{-1}$ can be identified with elements of $\operatorname{Symp}_{c}\left(\mathbb{R} P^{2}\right)$ and this implies $t \cdot u^{-1} \in H$, hence $t \in H$ up to homotopy.

Now we can finally complete the proof of Lemma 4.4. Combining lemmata above, we have the following homotopy fibration:

$$
H \hookrightarrow \operatorname{Stab}_{c}^{\text {Iso }}\left(C_{4}\right) \longrightarrow S^{1} .
$$

Since, by Lemma $4.8, \operatorname{Stab}_{c}^{\text {Iso }}\left(C_{4}\right)$ is weakly contractible, we deduce that $H$ is weakly homotopy equivalent to $\mathbb{Z}$ and, by Lemma 4.2 , that $\pi_{0}(H) \cong \mathbb{Z}$. This concludes our proof of Lemma 4.4.

Remark 4.11. There is an interesting model describing the action of $H$ in $U$ communicated to the authors by Yi Liu. Consider $\mathbb{R}^{3}$ with the standard Euclidean metric $g_{E}$. Consider an oriented normal frame $\left(e_{1}, e_{2}, e_{3}\right)$ as a point on $\mathbb{R} P^{3}$, it fibers over $S^{2}$ by projection to $e_{1}$. Let $\varpi: \mathbb{R} P^{3} \rightarrow \mathbb{R} P^{3}$ be the involution sending $\left(e_{1}, e_{2}, e_{3}\right)$ to $\left(-e_{1},-e_{2}, e_{3}\right)$ and consider its quotient $L(1,4)$. This can be identified with the unit cotangent bundle of $\mathbb{R} P^{2}$ and 
fibers over $\mathbb{R} P^{2}$ by the projection

$$
\left[e_{1}, e_{2}, e_{3}\right] \rightarrow\left[e_{1}\right]
$$

with fiber $S^{1}$. On the other hand, one may project $L(1,4)$ to $S^{2}$ by sending

$$
\left[e_{1}, e_{2}, e_{3}\right] \rightarrow e_{3}
$$

Endow all the spaces involved with the metric inherited by $g_{E}$ and use the obvious $S O(3)$ action on $\mathbb{R} P^{2}$ as constructed, then the projections interplay correctly with the symplectic structure on $T^{*} \mathbb{R} P^{2}$. In other words, given an isometry of $\mathbb{R} P^{2}$, represented by an element $R \in S O(3)$ in the above model, the corresponding action on the unit cotangent bundle is described by the same element $R$ acting on $L(1,4)$. In turn $R$ acts on the fibration (4.5). In this way we retrieve the action of $\left.H\right|_{U}$.

Proof of Theorem 1.3: For $\pi_{i}(\mathcal{L}), i \geq 1$ the theorem follows immediately from Lemma 4.4 and the homotopy fibration (4.1). Since the Dehn twists are also contained in the subgroup $\operatorname{Stab}_{c}^{\text {Iso }}(\mathbf{0})$, one sees that the map $\pi_{0}\left(\operatorname{Stab}_{c}(\mathbf{0})\right)$ $\rightarrow \pi_{0}\left(\operatorname{Symp}_{c}\left(T^{*} \mathbb{R} P^{2}\right)\right)$ is surjective. However, by Lemma 4.2, since both groups are $\mathbb{Z}$, it can only be an isomorphism.

\section{Appendix A. The diffeomorphism group of $\mathbb{R} \boldsymbol{P}^{2}$}

We give a proof of Proposition 4.3:

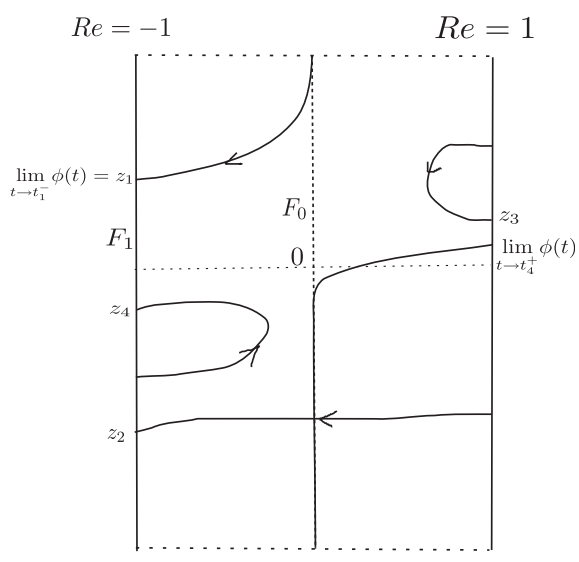

(a) "Example" of a curve in $\mathcal{F}_{2}$

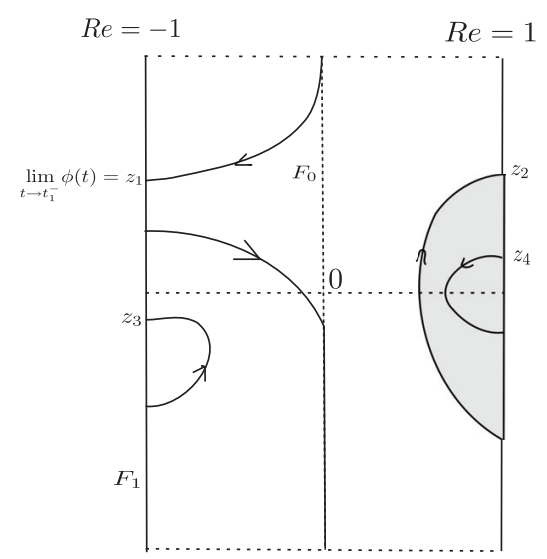

(b) Pushing the curve 
Proof. Thinking of $\mathbb{R} P^{2}=S^{2} / \sim$, where the equivalence relation identifies antipodal points, the action of $S O(3)$ on $S^{2}$ preserves equivalence classes and thus descends to an action on $\mathbb{R} P^{2}$. Therefore $\operatorname{Diff}\left(\mathbb{R} P^{2}\right)$ contains $S O(3)$ as a subgroup. We will show that the homogeneous space $\operatorname{Diff}\left(\mathbb{R} P^{2}\right) / S O(3)$ is weakly contractible. Fix an $x \in \mathbb{R} P^{2}$ and a frame $p$ at $x$. The diffeomorphism group $\operatorname{Diff}\left(\mathbb{R} P^{2}\right)$ is homotopic to the subset of maps which act as an isometry on $T_{x} \mathbb{R} P^{2}$. Given such an $f \in \operatorname{Diff}\left(\mathbb{R} P^{2}\right)$, there exists a unique element $\iota_{f} \in$ $S O(3)$, such that $\iota_{f} \circ f$ fixes $p$, and up to homotopy we may assume it fixes a neighborhood of $x$. Here we note that there are two disjoint circles in $S O(3)$ which map $f(x)$ onto $x$, but only elements in one compose with $f$ to give an orientation preserving map of $T_{x} \mathbb{R} P^{2}$, and of those there is a unique element in the circle which fixes the frame. Therefore, as the complement of a ball in $\mathbb{R} P^{2}$ is a Möbius band, we may identify $\operatorname{Diff}\left(\mathbb{R} P^{2}\right) / S O(3)$ with the compactly supported diffeomorphism group of the Möbius band $B$ with the boundary removed, which we denote as $\operatorname{Diff}_{c}(B)$. This is homotopic to the diffeomorphisms of the closed Möbius band which fix the boundary. Below, we identify $B$ with the bundle $\pi: B \rightarrow S^{1}$ with fibers the unit interval.

We fix a fiber $F_{0}$ over $p_{0} \in S^{1}$ and parameterize $F_{0}$ as a map

$$
F_{0}:(-\infty,+\infty) \rightarrow B
$$

Define

$$
\begin{aligned}
\mathcal{F}=\left\{\phi:(-\infty, \infty) \rightarrow B: \phi(t)=F_{0}(t)\right. & \text { when }|t|>R \\
& \text { for some } R, \phi \text { is embedded }\} .
\end{aligned}
$$

Then for $\phi \in \mathcal{F}$ we have that $\pi \circ \phi$ is a closed loop in $S^{1}$ with a well defined degree. Given this, we partition $\mathcal{F}$ as follows:

$$
\mathcal{F}_{i}=\{\phi \in \mathcal{F}: \operatorname{deg}(\pi \circ \phi)=i\}
$$

Lemma A.1. $\mathcal{F}_{i}$ is connected when $i=0$, and empty except when $i=$ $-1,0,1$. Curves in $\mathcal{F}_{1,-1}$ divide $B$ into two components.

Proof. Consider the strip $I=\{|\operatorname{Re}(z)| \leq 1\}$ in $\mathbb{C}$, then $B$ is obtained by gluing the two edges of the strip by $z \sim(-z)$ if $|R e(z)|=1$ (see Figure (a)). We denote the distinguished fiber in $B$ obtained from the glued edges by $F_{1}$. For a curve $\phi \in \mathcal{F}$, we may assume that it intersects $F_{1}$ transversely. We thus have a finite subset $T$ of $\mathbb{R}$, such that $T=\phi^{-1}\left(F_{1}\right)$. Write $T=\left\{t_{i}\right\}$ where the $t_{i}$ are in increasing order. 
Formally, we now consider $\phi$ as a map $\phi: \mathbb{R} \backslash T \rightarrow \stackrel{\circ}{I}$, to the interior of $I$ such that

$$
\lim _{t \rightarrow t_{i}^{+}} \phi(t)=-\lim _{t \rightarrow t_{i}^{-}} \phi(t) .
$$

Let $z_{i}=\phi\left(t_{i}\right)=\lim _{t \rightarrow t_{i}^{-}} \phi(t)$. For $\phi \in \mathcal{F}_{i}$ with $i \neq 0, T$ must be non-empty.

Claim. There exists an isotopy of $\phi$ to a curve $\phi^{\prime} \in \mathcal{F}$ with corresponding points $z_{j}^{\prime}$ such that either $\operatorname{Re}\left(z_{j}^{\prime}\right)=-1$ or $\operatorname{Re}\left(z_{j}^{\prime}\right)=1$ for all $j$.

Proof of Claim. If $\operatorname{Re}\left(z_{i}\right)=-\operatorname{Re}\left(z_{i+1}\right)$ for some $i$ then the image of $\left.\phi\right|_{\left(t_{i}, t_{i+1}\right)}$ is a curve in $\stackrel{\circ}{I}$ converging at both ends to points on the same edge. It is possible that the region formed by $\left.\phi\right|_{\left(t_{i}, t_{i+1}\right)}$ and $F_{1}$ contains other such loops (see the shaded area of Figure (b)). If there are no such loops then the region is empty. Hence we can find a $j$ such that $\operatorname{Re}\left(z_{j}\right)=-\operatorname{Re}\left(z_{j+1}\right)$ and the region formed by $\left.\phi\right|_{\left(t_{i}, t_{i+1}\right)}$ and $F_{1}$ is empty. Now we can perform an isotopy to remove the intersections $z_{j}$ and $z_{j+1}$ by pushing $\phi$ across the region. After such an isotopy the number of intersection points with $F_{1}$ will reduce by 2 and so after a finite number we must arrive at a curve satisfying our claim.

Given a curve $\phi$ we may now assume that $\operatorname{Re}\left(z_{i}\right)=\operatorname{Re}\left(z_{j}\right)$ for all $i, j$. Without loss of generality suppose that $\operatorname{Re}\left(z_{i}\right)=1$ for all $i$. Then if $\phi \in \mathcal{F}_{0}$ we see that $\phi$ avoids $F_{1}$ completely and thus is isotopic to $F_{0}$. This proves the first statement.

For the second statement, assume that $|T| \geq 2$, that is, there are intersections $z_{1}$ and $z_{2}$ with $T_{1}$. Then we observe that all paths $\left.\phi\right|_{\left(t_{i}, t_{i+1}\right)}$ must lie beneath $\left.\phi\right|_{\left(t_{1}, t_{2}\right)}$ for all $i \geq 2$, and thus cannot converge towards $+\infty$ in $I$. This gives a contradiction thus proving the second statement. The final statement is similarly clear.

Corollary A.2. The space $\operatorname{Diff}_{c}(B)$ is connected.

Proof. Indeed, any $f \in \operatorname{Diff}_{c}(B)$ maps $F_{0}$ to a path which, like $F_{0}$ cannot divide $B$. Thus, by Lemma A.1 the image of $F_{0}$ lies in $\mathcal{F}_{0}$ and, moreover, we may assume up to isotopy that $f$ fixes $F_{0}$, and by a further isotopy a neighborhood of $F_{0}$ and the complement of a compact set in $B$. But removing a tubular neighborhood of the boundary and $F_{0}$ from $B$ leaves a set diffeomorphic to a disk, and as the group of diffeomorphisms of the disk is connected, see [21], our corollary follows.

Recall that to prove Proposition 4.3 we must show that $\operatorname{Diff}_{c}(B)$ is contractible. Line fields on $B$ are maps from $B$ to its projectivized unit tangent 
bundle, where we identify vectors differing up to sign. We will only consider fields which are trivial, that is, coincide with fibers of $B$, outside of a compact set. The bundle is trivialized by the fibers of $B$ and so line fields are equivalent to maps from $B$ to $\mathbb{R} P^{1}$. Let $l_{0}$ be the trivial line field tangent to the fibers. The space of sections $\mathcal{L}_{0}$ homotopic to $l_{0}$ (through trivial line fields) is contractible as all such sections lift to maps to $\mathbb{R}$ with compact support.

We now make some additional observations about the line fields in $\mathcal{L}_{0}$, eventually concluding that they have no closed loops.

Claim 1: Closed loops in a line field in $\mathcal{L}_{0}$ project to $S^{1}$ with degree 1 or 2 (up to sign).

Proof of Claim 1: There exists a double cover from an annulus $A \rightarrow B$ and so line fields in $\mathcal{L}_{0}$ lift to line fields on $A$. As the line field is never 0 , any closed loops in $A$ cannot bound a compact region. Therefore they must represent a nontrivial homotopy class, and actually a generator since the loops are embedded. Projecting back to $B$, under the projection $B \rightarrow S^{1}$ the closed loop must have degree 1 (if it is a double cover of a loop in $A$ ) or 2 , up to sign.

Claim 2: If a line field in $\mathcal{L}_{0}$ has a closed loop of degree 2 then it also has a closed loop of degree 1 .

Proof of Claim 2: By an argument similar to that in the proof of Lemma A.1 we see that a closed loop of degree 2 must bound a compact region $G$ in $B$, and up to an isotopy we may assume that $G \cap F_{0}$ is an interval. There is a return map from $G \cap F_{0}$ to itself given by following the line field, and this map reverses the two boundary points. Thus the return map must have a fixed point, and this corresponds to a loop of degree 1.

Claim 3: A line field in $\mathcal{L}_{0}$ has no closed loops of degree 1.

Proof of Claim 3: We will show that the set of line fields $\mathcal{K} \subset \mathcal{L}_{0}$ with a closed loop of degree 1 are both open and closed. Therefore since $\mathcal{L}_{0}$ is connected either all line fields have such a closed loop or none do, and as $l_{0}$ certainly has no closed loop this will complete the proof.

To see that $\mathcal{K}$ is open, we observe that the Poincaré return map defined on a suitable interval transverse to a closed loop of degree 1 is orientation reversing, and so the fixed point is stable.

To see that $\mathcal{L}_{0} \backslash \mathcal{K}$ is open, we observe that if a line field has no closed loops then every integral curve must converge to $\partial B$ in both forward and backward time, and by definition coincides with trivial fibers outside of a 
compact set. As solutions of ODEs depend continuously on parameters, this property is preserved under small perturbations of the line field.

Now, starting with a line field in $\mathcal{L}_{0}$, the above claims imply that there are no closed loops and so all integral curves coincide with fibers of $B$ outside of a compact set (although each end of a curve may correspond to a different fiber). Thus, following these curves we get a orientation preserving diffeomorphism from $S^{1}$ (thought of as the boundary of $B$ ) to itself with the following properties: it does not have any fixed points, and squares to identity.

Claim: The space of such diffeomorphisms, denoted as D, is contractible.

Proof of the Claim: Fix a point $x_{0} \in S^{1}$. Given $f \in D$, consider $f\left(x_{0}\right)$ which lies in the contractible set $S^{1} \backslash\left\{x_{0}\right\}$. Such assignment $D \rightarrow S^{1} \backslash\left\{x_{0}\right\}$ is clearly a fibration.

For any choice of $f\left(x_{0}\right)$, the two points $x_{0}$ and $f\left(x_{0}\right)$ divide $S^{1}$ into two closed intervals $I_{1}$ and $I_{2}$ (including these two points themselves). Therefore, $f$ is identified with a diffeomorphism from $I_{1}$ to $I_{2}$ since it is orientation preserving. Such diffeomorphisms are further identified with $\operatorname{Diff}\left(I_{1}\right)$, which is also contractible. (Thinking of the diffeomorphisms as graphs on the interval, it is a convex set.) The claim then follows.

Notice that deformations in $D$ can be generated by deformations of the line fields near the boundary of $B$. Therefore there is a deformation retract from $\mathcal{L}_{0}$ to line fields whose integral curves coincide with the same fiber outside of a compact set. Up to a choice of parameterizing the curves, such line fields generate elements of $\operatorname{Diff}_{c}(B)$ by mapping the fibers onto the corresponding integral curves and hence, up to homotopy, we get a natural $\operatorname{map} \mathcal{L}_{0} \rightarrow \operatorname{Diff}_{c}(B)$.

We now complete the proof of Proposition 4.3. Given an $f \in \operatorname{Diff}_{c}(B)$ the line field $f_{*} l_{0}$ is homotopic to $l_{0}$ by Corollary A.2. Thus we have a continuous map $\operatorname{Diff}_{c}(B) \rightarrow \mathcal{L}_{0}$. (Recall that $\mathcal{L}_{0}$ consists of line fields homotopic to $l_{0}$.) By definition it is a homotopy inverse to the map above and hence $\operatorname{Diff}_{c}(B)$ is homotopic to $\mathcal{L}_{0}$, which is contractible, and the proof is complete.

\section{References}

[1] M. Abreu and D. McDuff, Topology of symplectomorphism groups of rational ruled surfaces, J. Amer. Math. Soc., 13 (2000), no. 4, 9711009. 
[2] S. Anjos and M. Pinsonnault, The homotopy Lie algebra of symplectomorphism groups of 3-fold blow-ups of the projective plane, Math. Zeitschrift, Published online 10 January 2013, 48 pages. arXiv: 1201.5077.

[3] M. Audin, On the topology of Lagrangian submanifolds, Examples and counter-examples, Portugaliae Mathematica, 62 (2005), 375-419.

[4] J. Borzellino and V. Brunsden, The Stratified Structure of Spaces of Smooth Orbifold Mappings, to appear in Comm. Contemporary Math., arXiv:0810 . 1070.

[5] J. Coffey, Symplectomorphism groups and isotropic skeletons, Geom. Topol., 9 (2005), 935-970.

[6] J. D. Evans, Lagrangian spheres in Del Pezzo surfaces, J. Topol., 3 (2010), no. 1, 181-227.

[7] J. D. Evans, Symplectic mapping class groups of some Stein and rational surfaces, J. Symplectic Geom., 9 (2011), no. 1, 45-82.

[8] J. Evans, Symplectic topology of some Stein and rational surfaces, Ph.D. thesis, University of Cambridge.

[9] A. Hatcher, Algebraic topology, Cambridge University Press, Cambridge, 2002. xii+544 pp.

[10] M. Gromov, Pseudo holomorphic curves in symplectic manifolds, Invent. Math., 82 (1985), no. 2, 307-347.

[11] R. Hind, Lagrangian spheres in $S^{2} \times S^{2}$. Geom. Funct. Anal., 14 (2004), no. $2,303-318$.

[12] R. Hind, Lagrangian unknottedness in Stein surfaces, Asian J. Math., 16 (2012), 1-36.

[13] R. Hind and A. Ivrii, Ruled 4-manifolds and isotopies of symplectic surfaces, Math. Z., 265 (2010), no. 3, 639-652.

[14] E. Lerman, Symplectic cuts. Math. Res. Lett., 2 (1995), no. 3, 247-258.

[15] D. McDuff, The structure of rational and ruled symplectic 4-manifolds. J. Amer. Math. Soc., 3 (1990), no. 3, 679-712.

[16] D. McDuff, Remarks on the uniqueness of symplectic blowing up, in: Symplectic Geometry; ed. by D. Salamon; London Math. Soc. Lecture Note Ser., 192, Cambridge Univ. Press, Cambridge, 1993, 157-67. 
[17] D. McDuff, From symplectic deformation to isotopy, Topics in symplectic 4-manifolds (Irvine, CA, 1996), 85-99, Int. Press Lect. Ser., I, Int. Press, Cambridge, MA, 1998.

[18] D. McDuff, D. Salamon, Introduction to symplectic topology, 2nd edition. Oxford Math. Mono. The Clarendon Press, Oxford University Press, New York, 1998.

[19] P. Seidel, Symplectic automorphisms of $T^{*} S^{2}$, arXiv:9803084.

[20] P. Seidel, Lectures on four-dimensional Dehn twists, Symplectic 4manifolds and algebraic surfaces, 231-267, LNM 1938, Springer, Berlin, 2008.

[21] S. Smale, Diffeomorphisms of the 2-sphere, Proc. Amer. Math. Soc., 10 (1959), 621-626.

[22] M. VanHoof, Symplectomorphism groups of weighted projective spaces and related embedding spaces, Ph.D. thesis, University of Western Ontario, 2013.

Department of Mathematics, University of Notre Dame

255 Hurley, Notre Dame, IN 46556, USA

E-mail address: Richard.K.Hind.1@nd.edu

Department of Mathematics, The University of Western Ontario

London, Ontario, Canada, N6A 5B7

E-mail address: mpinson@uwo.ca

Department of Mathematics, 452 Boyd Graduate Studies

University of Georgia, Athens, GA 30602, USA

E-mail address: mathwww@gmail.com

RECEIVED July 19, 2013 